Potentially Missing Physics of the Early Universe: Nonlinear Vacuum Polarization in Intense Blackbody Radiation

S.S.Q. Wu, F.V. Hartemann

April 14, 2010 
This document was prepared as an account of work sponsored by an agency of the United States government. Neither the United States government nor Lawrence Livermore National Security, LLC, nor any of their employees makes any warranty, expressed or implied, or assumes any legal liability or responsibility for the accuracy, completeness, or usefulness of any information, apparatus, product, or process disclosed, or represents that its use would not infringe privately owned rights. Reference herein to any specific commercial product, process, or service by trade name, trademark, manufacturer, or otherwise does not necessarily constitute or imply its endorsement, recommendation, or favoring by the United States government or Lawrence Livermore National Security, LLC. The views and opinions of authors expressed herein do not necessarily state or reflect those of the United States government or Lawrence Livermore National Security, LLC, and shall not be used for advertising or product endorsement purposes.

This work performed under the auspices of the U.S. Department of Energy by Lawrence Livermore National Laboratory under Contract DE-AC52-07NA27344. 


\title{
Potentially Missing Physics of the Early Universe: Nonlinear Vacuum Polarization in Intense Blackbody Radiation
}

\author{
Sheldon S.Q. Wu and Frederic V. Hartemann
}

Table of Contents

\begin{tabular}{ll} 
TABLE OF CONTENTS & 1 \\
\hline
\end{tabular}

PROJECT DESCRIPTION

I. INTRODUCTION

II. PROJeCt PLAN

III. MANAgement PLAN 5

IV. DISSEMINATION

V. SUMMARY

REFERENCES CITED

BIOGRAPHICAL SKETCHES 8




\section{Project Description}

\section{Introduction}

The standard Big Bang universe model is mainly based on linear interactions, except during exotic periods such as inflation. The purpose of the present proposal is to explore the effects, if any, of vacuum polarization in the very high energy density environment of the early universe. These conditions can be found today in astrophysical settings and may also be emulated in the laboratory using high intensity advanced lasers.

Shortly after the Big Bang, there once existed a time when the energy density of the universe corresponded to a temperature in the range $10^{8}-10^{9} \mathrm{~K}$, sufficient to cause vacuum polarization effects. During this period, the nonlinear vacuum polarization may have had significant modifications on the propagation of radiation. Thus the thermal spectrum of the early universe may have been starkly non-Planckian. Measurements of the cosmic microwave background today show a spectrum relatively close to an ideal blackbody. Could the early universe have shown spectral deviations due to nonlinear vacuum effects? If so, is it possible to detect traces of those relic photons in the universe today?

Found in galactic environments, compact objects such as blazars and magnetars can possess astronomically large energy densities that far exceed anything that can be created in the laboratory. Their field strengths are known to reach energy levels comparable to or surpassing the energy corresponding to the Schwinger critical field $\mathrm{E} \sim 10^{18} \mathrm{~V} / \mathrm{m}$. Nonlinear vacuum effects become prominent under these conditions and have garnered much interest from the astronomical and theoretical physics communities. The effects of a nonlinear vacuum may be of crucial importance for our understanding of these objects.

At energies of the order of the electron rest mass, the most important interactions are described by quantum electrodynamics (QED). It is predicted that nonlinear photon-photon interactions will occur at energies approaching the Schwinger critical field. The basic process is the appearance of vacuum polarization, or the creation of the virtual electron-positron pair by vacuum fluctuations, as shown in Fig.1. These quantum processes can be described by an effective field theory for the electromagnetic field where the effects of virtual processes appear as small corrections. First derived by Heisenberg and Euler, this theory describes the corrections to classical electromagnetic theory due to photon-photon scattering [1]. An overview of nonlinear vacuum effects as formulated through the Heisenberg-Euler Lagrangian can be found in [2]. 


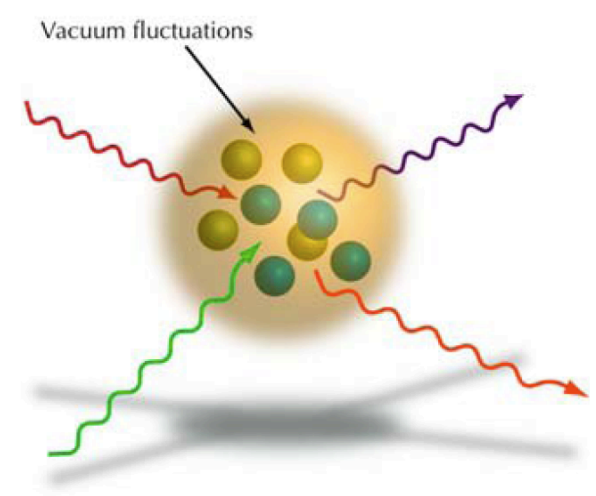

Fig. 1 The process of elastic scattering among photons through the quantum vacuum. The wavy lines represent photons and spheres represent virtual electrons and positrons. Artwork from

Eur. Phys. J. D 55, 319-326 (2009)

\section{Project Plan}

We propose a novel theoretical study to pursue a fully self-consistent theory of blackbody radiation in the high energy density regime. We plan to accomplish this by building upon the existing effective-field theory using the Heisenberg-Euler Lagrangian, in conjunction with the framework of stochastic electrodynamics (SED) and thermodynamics to fold the effects of the nonlinear QED vacuum into a semi-classical model of the blackbody.

From an electromagnetic radiation viewpoint, inertially-confined ignited deuteriumtritium plasmas, similar to that produced in the National Ignition Facility (NIF), behave rather like blackbodies, with characteristic temperatures of a few tens of keV [3]. It has been shown that the injection of relativistic electrons into the burning core can generate high energy photons via Compton scattering. While the thermal energy density of the core will correspond to field strengths much lower than the Schwinger field necessary to produce photon-photon scattering, it has been speculated that electron focusing could be used to focus the high energy photons in a very small spatial region, where the electromagnetic fields may approach or exceed the Schwinger limit [4]. Due to the Laboratory's dedication toward nuclear and energy security, lead by the NIF and followed by the upcoming Laser Inertial Confinement FusionFission Energy (LIFE) program, we expect that a physical model and numerical code that includes vacuum polarization effects in the presence of blackbody radiation will greatly enhance the Laboratory's modeling capabilities.

Owing to enormous progress in the field of high-power laser facilities, today the brightest lasers are capable of reaching intensities in excess of $10^{22} \mathrm{~W} / \mathrm{cm}^{2}$. At these intensities, the nonlinear relativistic dynamics of laser-plasma systems give rise to numerous high field effects, including high harmonic generation, soliton formation and pulse collapse. At higher 
intensities, the combined effects of laser pulse collapse and ponderomotive force electron expulsion can create conditions in which the nonlinear vacuum effect of elastic photon-photon scattering and electron-positron creation become important. Simple experiments have already been suggested for measuring these effects in upcoming high power laser facilities like the European Extreme Light Infrastructure (ELI) project [5]. The physical understanding and numerical modeling of laser-plasma interactions at these intensities is vital to the next generation of laser experiments, including the upcoming LIFE program here at Livermore.

While incoherent blackbody radiation is at one end of the scale, and coherent photon field of the laser is at another, there are similar aspects of the theory that apply to both. Computer modeling codes that result from the proposed work can benefit a wider scientific community. One potentially fruitful numerical model is a Monte-Carlo simulation of intense blackbody radiation and its interaction with photons and particles. This code will be very helpful in understanding the interaction dynamics and, ultimately, the early universe. The Laboratory's renowned supercomputing infrastructure may be utilized in the second year of this study, when a working prototype computer code is expected, to help achieve this objective.

In previous works (e.g. [6]), the effective nonlinear Lagrangian derived by Heisenberg and Euler is used to describe the propagation of photons in slowly varying but otherwise arbitrary electromagnetic fields. One can extend these results to collections of fields by aggregating the interactions of individual components. However, since the principle of superposition does not hold for nonlinear systems, approaches based on conventional methods of solving linear differential equations may be inappropriate. For a statistical mixture of many interacting fields, one needs to examine all of the fields collectively.

One method to approach this problem is via stochastic electrodynamics, which is well suited to describing the random nature of thermal fields. In SED, both the fields and particles with which it interacts are treated classically. SED has been successfully applied to a number of non-classical problems in physics, including zero-point energy, the Casimir force and the UnruhDavies effect for accelerated observers $[7,8]$. To the best of our knowledge, SED has not yet been applied in the scope of nonlinear vacuum polarization. We believe SED to be a useful framework on which we can build upon to include nonlinear vacuum polarization into high energy density photon thermodynamics.

The deliverables in this proposed study are:

A. Theoretical models and computer codes applicable to high intensity lasers, astrophysics, high energy density physics, NIF and LIFE.

B. A series of scientific publications in professional journals 
1. Novel derivation of lowest order QED corrections to photon thermodynamics and blackbody radiation -Physical Review Letters, 2010

2. Study of the radiation dynamics of charged particles in intense blackbody fields with application to the Cherenkov effect -JOSA B or Optics Letters 2010-2011

3. A self-consistent theory of high energy density blackbody radiation -Journal of Physics A or Physical Review D, 2011-2012

4. Nonlinear vacuum polarization and the Unruh effect -Phys Rev Lett, 2011-2012

C. Conference presentations and proceedings (details in Section IV)

\section{Management Plan}

We are asking for support at the $\$ 225 \mathrm{~K}$ level and a period of 2 years to complete this theoretical study. We are proposing that this study be lead by Dr. Sheldon Wu, a former Lawrence Scholar and now a post-doctoral researcher in NIF/PS\&A. Dr. Frederic Hartemann, a chief scientist in NIF/PS\&A, will provide additional support and consultation. The proposed numerical portion of this study will be aided in the second year, if necessary, by institutional computation capabilities that are available for general use. Finally, the PS\&A staff that will contribute to this work, along with their NIF/PS\&A management, have an established record of successfully completing projects, reporting/publishing their results in the scientific literature, and building follow-on programs that show a return on investment of the LLNL supported R\&D. We expect this theoretical and numerical study to lead to further research after the LDRD funding period and potentially gain favorable recognition from other institutions and funding agencies.

\section{Dissemination}

The proposed work will result in a number of publications in journals, professional conferences, technical seminars, etc. If successful, we expect this theoretical study to lead to collaborative efforts with other scientists inside and outside of the Laboratory. To help engender this outcome, travel to technical or scientific conferences is an integral part of this plan. Appropriate conferences to disseminate our results are:

-American Physics Society, General Meetings, March \& April 2011

-American Physics Society, Division of Plasma Physics, Nov 2010

-American Astronomical Society, January \& June 2011

-American Astronomical Society, High Energy Astrophysics Division, March 2011 


\section{Summary}

The purpose of the present proposal is to explore the effects, if any, of the nonlinear vacuum in the very high energy density environment of the early universe. This investigation will lead to a novel description of the propagation of light and interaction with matter in the nonlinear high energy density vacuum. Our conjecture is supported by a number of works in literature from disparate branches of physics. Our results may be applicable to a wide array of fields, including high intensity lasers, astrophysics and cosmology, high energy density physics and fusion. This activity is inline with LLNL's strategic roadmap and will contribute to maintaining LLNL's scientific vitality and world eminence in the field of fundamental physics and astrophysics, in addition to enhancing the Laboratory's ability to address future high energy density strategic missions, including but not limited to NIF and LIFE. 


\section{References Cited}

[1a] W. Heisenberg, H. Euler, "Folgerungen aus der Diracschen Theorie des Positrons", Zeitschr. Phys. 98, 714 (1936)

[1b] W. Heisenberg, H. Euler, "Consequences of Dirac Theory of the Positron", translated by W. Korolevski and H. Kleinert, FU-Berlin, arXiv:physics/0605038v1

[2] M. Marklund, P.K. Shukla, "Nonlinear collective effects in photon-photon and photonplasma interactions", Rev. Mod. Phys. 78, 591-640 (2006)

[3] J. Lindl, "Development of the indirect-drive approach to inertial confinement fusion and the target physics basis for ignition and gain," Phys. Plasmas 2, 3933-4024 (1995)

[4] F. V. Hartemann, C. W. Siders, and C. P. J. Barty, "Theory of Compton scattering in ignited thermonuclear plasmas," J. Opt. Soc. Am. B 25, B167-B174 (2008)

[5] D. Tommasini, A. Ferrando, H. Michinel, M. Seco, "Detecting photon-photon scattering in vacuum at exawatt lasers", Phys. Rev. A 77, 042101 (2008)

[6] Z. Bialynicka-Birula, I. Bialynicki-Birula, "Nonlinear Effects in Quantum Electrodynamics. Photon Propagation and Photon Splitting in an External Field", Phys. Rev. D 2, 2341-2345 (1970)

[7] T.H. Boyer, "Derivation of the Blackbody Radiation Spectrum without Quantum Assumptions", Phys. Rev. 182, 1374-1383 (1969)

[8] T.H. Boyer, "Thermal effects of acceleration through random classical radiation", Phys. Rev. D 21, 2137-2148 (1980) 


\title{
Biographical Sketches
}

\section{Sheldon S.Q. Wu}

\author{
Postdoctoral researcher
}

$925-423-7732$

wu31@IInl.gov

\section{(a) Professional Preparation}

A list of the individual's undergraduate and graduate education and postdoctoral training as indicated below:

University of California, San Diego Electrical Engineering $\quad$ B.S. 2004

University of California, San Diego Electrical Engineering Ph.D. 2009

\section{(b) Appointments}

Postdoctoral appointment at LLNL from 2009-2011

\section{(c) Awards}

Lawrence Scholar recipient in the Lawrence Scholar Program, 2008-2009

Graduate Scholarship recipient in 2007-2008 and 2008-2009 awarded by the High Energy Laser Joint Technology Office and the Directed Energy Professional Society (DEPS)

NIF Directorate Performance Award, 2007

\section{(d) Publications}

i. S. S. Q. Wu, T. F. Soules, R. H. Page, S. C. Mitchell, V. Keith Kanz, and R. J. Beach, "Hydrocarbon-free resonance transition 795-nm rubidium laser," Opt. Lett. 32, 2423-2425 (2007)

ii. S. S. Q. Wu, T. F. Soules, R. H. Page, S. C. Mitchell, V. Keith Kanz, and R. J. Beach, "Resonance transition 795-nm rubidium laser using ${ }^{3} \mathrm{He}$ buffer gas", Optics Communications, Vol 281, 5, 1222-1225, 2008

iii. S. S. Q. Wu, T. F. Soules, R. H. Page, S. C. Mitchell, V. Keith Kanz, and R. J. Beach, "Resonance transition 795-nm Rubidium laser with He buffer gas" (Invited paper), Proc. SPIE, Vol. 7005, 700523 (2008)

iv. S. S. Q. Wu, F. V. Hartemann, C.W. Siders, and C. P. J. Barty, American Physical Society, 51st Annual Meeting of the APS Division of Plasma Physics, November 2-6, 2009, abstract \#GP8.090

v. S. S. Q. Wu, F. V. Hartemann, and C. P. J. Barty, 11th High Energy Astrophysics Division (HEAD) Program, Bulletin of the American Astronomical Society (BAAS) volume 42 \#2, 2010 


\section{Frederic V. Hartemann}

LLNL B482 R1246

7000 East Avenue, Livermore, CA 94550

(925) 423-3398

hartemann1@IInl.gov

\section{(a) Professional Preparation}

U. Paris XI Physics BS 1980

U. Paris XI Physics PhD 1986

$\begin{array}{lll}\text { MIT } & \text { FELS } & 1987\end{array}$

\section{(b) Appointments}

LLNL

University of California

MIT - Visiting Scientist

Thomson Electron Tubes
2001

1993-2001

1988-1991

1987-2001

\section{(d) Publications}

High-field electrodynamics, CRC Press (2000)

Phys. Rev. Lett. 59, 1177 (1987)

Phys. Rev. Lett. 72, 1192 (1994)

Phys. Rev. Lett. 72, 2391 (1994)

Phys. Rev. Lett. 74, 1107 (1995)

Phys. Rev. Lett. 76, 624 (1996)

Phys. Rev. Lett. 100, 125001 (2008)

Phys. Rev. STAB 7, 060702 (2004)

Phys. Rev. STAB 8, 100702 (2005)

Phys. Rev. STAB 10, 011301 (2007) 